\title{
K-type slant helices on spacelike and timelike surfaces
}

\author{
Santosh Kumar and Buddhadev Pal
}

\begin{abstract}
We have derived a necessary and sufficient condition for a non-null normal spacelike curve lying in a spacelike or a timelike surface $M \subset E_{1}^{3}$, so that the curve becomes a $K$-type spacelike slant helix with $K \in\{1,2,3\}$. We have used Darboux frame to define necessary and sufficient conditions. An example is given for a 1-type spacelike slant helix having a spacelike normal and a timelike binormal.
\end{abstract}

\section{Introduction}

A curve $\gamma$ in Euclidean space whose tangent vector makes a constant angle with some fixed direction is known as a general helix. The necessary and sufficient condition, the ratio of non zero curvature and torsion of general helix is constant. The condition was first defined by M. A. Lancret in 1802 and the first proof for the condition was given by B. de Saint Venant in 1845. A new version of proof by using Killing vector field for the condition was given in [5]. Izumiya and Takeuchi [6] have introduced the concept of a slant helix in Euclidean space. They have defined a slant helix as a curve whose principal normal makes a constant angle with some fixed direction. A unit speed curve $\gamma$ is a slant helix if and only if the function

$$
K(s)=\left(\frac{\kappa^{2}}{\left(\kappa^{2}+\tau^{2}\right)^{\frac{3}{2}}}\left(\frac{\tau}{\kappa}\right)^{\prime}\right)(s), \quad \kappa(s) \neq 0,
$$

is constant. The curve of constant precession is a slant helix and the spherical image of the tangent indicatrix (binormal indicatrix) for slant helix $\gamma$ is a spherical helix [8]. Position vector for a slant helix on $E^{3}$ was calculated

Received January 25, 2021.

2020 Mathematics Subject Classification. 53C50, 53C40.

Key words and phrases. Spacelike curve, spacelike slant helices, spacelike surface, Darboux frame.

https://doi.org/10.12697/ACUTM.2021.25.14

Corresponding author: Buddhadev Pal 
in [1], and the authors have proposed a condition that a unit speed curve $\gamma$ is a slant helix if and only if

$$
\tau(s)= \pm \frac{m k(s) \int \kappa(s) d s}{\sqrt{1-m^{2}\left(\int \kappa(s) d s\right)^{2}}},
$$

where $m=\frac{n}{\sqrt{1-n^{2}}}$ and $n=\cos \phi$ ( $\phi$ is the angle between a fixed line and the normal of the curve).

In 2011 Ali and Rafael [3] defined a slant helix in Minkowski space. A unit speed curve $\gamma$ in $E_{1}^{3}$ is said to be a slant helix if there exists a constant fixed direction $U$, whose inner product with principal normal is constant, i.e, $\langle N, U\rangle$ is constant, where $N$ is a principal normal for curve $\gamma$. A curve in Minkowski space may be spacelike, timeline, or lightlike according to this causal character of the curve. Thus, we get a condition for a curve to be a slant helix in the Minkowski space. A unit speed timelike curve in Minkowski space is a slant helix if and only if one of the following two functions is constant:

$$
G(s)=\left(\frac{\kappa^{2}}{\left(\tau^{2}-\kappa^{2}\right)^{\frac{3}{2}}}\left(\frac{\tau}{\kappa}\right)^{\prime}\right)(s), H(s)=\left(\frac{\kappa^{2}}{\left(\kappa^{2}-\tau^{2}\right)^{\frac{3}{2}}}\left(\frac{\tau}{\kappa}\right)^{\prime}\right)(s), \quad \tau^{2}-\kappa^{2} \neq 0 .
$$

Condition for a unit speed spacelike curve with spacelike normal to become a slant helix is the same as the condition defined for a timelike curve. But, if the normal of a spacelike curve is timelike, then the curve is a slant helix if and only if the function $K(s)$ is constant. Whereas every spacelike curve with lightlike normal is a slant helix. Position vector for a timelike slant helix in Minkowski space was studied in [4]. The concept of a $K$-type slant helix in $E_{1}^{4}$ was introduced in [2] and a $K$-type slant helix was defined in [2], using the Frenet frame, while we did the calculation for $K$-type spacelike slant helix by using Darboux frame. The relation between the Darboux frame and Frenet frame was defined in [7, 13]. Darboux helix (curve of constant precession) is an advanced version of a slant helix. A curve $\gamma$ whose Darboux vector $(D)$ makes a constant angle with a fixed direction vector $(V)$ is called a Darboux helix. Darboux helix in Euclidean space $E^{3}$ has been studied by Ziplar et al. [15]. To learn more about Darboux helix in Minkowski space we refer the reader to $[10,15,11]$.

Getting motivation from the above papers we have organized our paper as follows. We define $K$-type spacelike slant helices on the spacelike and timelike surfaces by using the Darboux frame. Then we define the conditions for 1-type, 2-type and 3-type spacelike slant helices and derive the relation between them. Also, we give an example of a 1-type spacelike slant helix on a spacelike surface which is neither 2-type nor 3-type spacelike slant helix. In the last section, we find the first axis for the Darboux helix using a Darboux frame which is orthogonal to the Darboux vector. 


\section{Preliminaries}

Minkowski 3-dimensional space $E_{1}^{3}$ is a real vector space $\mathbb{R}^{3}$ equipped with the metric

$$
\langle x, y\rangle=-x^{1} y^{1}+x^{2} y^{2}+x^{3} y^{3},
$$

where $x=\left(x^{1}, x^{2}, x^{3}\right)$ and $y=\left(y^{1}, y^{2}, y^{3}\right) \in \mathbb{R}^{3}$. Let $\gamma:[a, b] \longrightarrow M \subset E_{1}^{3}$ be a curve parametrised by the arc length parameter $s$. Then the curve $\gamma$ is said to be spacelike if $\left\langle\gamma^{\prime}(s), \gamma^{\prime}(s)\right\rangle=1$, timelike if $\left\langle\gamma^{\prime}(s), \gamma^{\prime}(s)\right\rangle=-1$, and lightlike if $\left\langle\gamma^{\prime}(s), \gamma^{\prime}(s)\right\rangle=0$.

Definition 1. A surface $M$ in $E_{1}^{3}$ is said to be spacelike if unit surface normal is a timelike vector and the surface $M$ is called a timelike surface if unit surface normal is a spacelike vector.

Let $\{T, N, B\}$ be the moving Frenet frame along a spacelike curve $\gamma$ parametrized by the arc length parameter $s$ lying in Minkowski space $E_{1}^{3}$, where $T, N, B$ are the tangent, the principal normal, and the binormal vector field along $\gamma$, respectively. Then the following cases can be studied according to the principal normal of curves (see [14]).

Case 1. If $\left\langle\gamma^{\prime \prime}(s), \gamma^{\prime \prime}(s)\right\rangle \neq 0$, then Frenet formulae for $\gamma$ are

$$
\left|\begin{array}{l}
T^{\prime} \\
N^{\prime} \\
B^{\prime}
\end{array}\right|=\left|\begin{array}{ccc}
0 & \kappa(s) & 0 \\
-\epsilon \kappa(s) & 0 & \tau(s) \\
0 & \tau(s) & 0
\end{array}\right|\left|\begin{array}{c}
T \\
N \\
B
\end{array}\right|,
$$

where $\langle N, N\rangle=\epsilon= \pm 1,\langle B, B\rangle=-\epsilon,\langle T, T\rangle=1,\langle T, N\rangle=\langle T, B\rangle=$ $\langle N, B\rangle=0$, and $T \times N=\epsilon B, N \times B=T, B \times T=\epsilon N$.

Case 2. If $\left\langle\gamma^{\prime \prime}(s), \gamma^{\prime \prime}(s)\right\rangle=0$, then Frenet formulae are given by

$$
\left|\begin{array}{l}
T^{\prime} \\
N^{\prime} \\
B^{\prime}
\end{array}\right|=\left|\begin{array}{ccc}
0 & \kappa(s) & 0 \\
0 & \tau(s) & 0 \\
-\kappa(s) & 0 & -\tau(s)
\end{array}\right|\left|\begin{array}{c}
T \\
N \\
B
\end{array}\right|,
$$

where $\langle N, N\rangle=\langle B, B\rangle=\langle T, B\rangle=\langle T, N\rangle=0,\langle T, T\rangle=1,\langle N, B\rangle=1$, and $T \times N=N, N \times B=T, B \times T=B$.

Darboux Frame. We know that the tangent vector field $\gamma^{\prime}(s)$ along a curve $\gamma$ lying on a surface $M$ is also the tangent vector field to the given surface $M$ along a curve $\gamma(s)$. If $Z$ is the unit surface normal at a point $\gamma(s)$ on the surface $M$, then we can construct a vector $Y= \pm T \times Z$. These three vectors $T, Y$, and $Z$ give rise to another moving frame along a curve on the surface $M$ known as the Darboux frame.

Spacelike surface. The relation between Darboux frame and Frenet frame for a spacelike curve with a spacelike normal lying on a spacelike 
surface is given as

$$
\left|\begin{array}{c}
T \\
Y \\
Z
\end{array}\right|=\left|\begin{array}{ccc}
1 & 0 & 0 \\
0 & \cosh (\theta) & \sinh (\theta) \\
0 & \sinh (\theta) & \cosh (\theta)
\end{array}\right|\left|\begin{array}{c}
T \\
N \\
B
\end{array}\right|,
$$

where $\langle T, T\rangle=1,\langle Z, Z\rangle=-1,\langle Y, Y\rangle=1$. After using equations (1) and (2) we can write that

$$
\left|\begin{array}{c}
T^{\prime} \\
Y^{\prime} \\
Z^{\prime}
\end{array}\right|=\left|\begin{array}{ccc}
0 & \kappa_{g} & \kappa_{n} \\
-\kappa_{g} & 0 & \tau_{g} \\
\kappa_{n} & \tau_{g} & 0
\end{array}\right|\left|\begin{array}{c}
T \\
Y \\
Z
\end{array}\right|,
$$

where $\langle T, T\rangle=\langle Y, Y\rangle=1,\langle Z, Z\rangle=-1, T \times Z=Y, Z \times Y=T, Y \times T=-Z$, and $\kappa_{g}=\kappa \cosh \theta, \kappa_{n}=\kappa \sinh \theta, \tau_{g}=\tau+\frac{d \theta}{d s}$.

The relation between Darboux frame and Frenet frame for a spacelike curve with a timelike normal lying on a spacelike surface is given by

$$
\left|\begin{array}{l}
T \\
Y \\
Z
\end{array}\right|=\left|\begin{array}{ccc}
1 & 0 & 0 \\
0 & \sinh (\theta) & \cosh (\theta) \\
0 & \cosh (\theta) & \sinh (\theta)
\end{array}\right|\left|\begin{array}{c}
T \\
N \\
B
\end{array}\right|,
$$

where $\langle T, T\rangle=1,\langle Z, Z\rangle=-1,\langle Y, Y\rangle=1$. After using equations (1) and (4) we can write

$$
\left|\begin{array}{c}
T^{\prime} \\
Y^{\prime} \\
Z^{\prime}
\end{array}\right|=\left|\begin{array}{ccc}
0 & \kappa_{n} & \kappa_{g} \\
-\kappa_{n} & 0 & \tau_{g} \\
\kappa_{g} & \tau_{g} & 0
\end{array}\right|\left|\begin{array}{c}
T \\
Y \\
Z
\end{array}\right|,
$$

where $\langle T, T\rangle=\langle Y, Y\rangle=1,\langle Z, Z\rangle=-1, T \times Z=Y, Z \times Y=T, Y \times T=-Z$, and $\kappa_{g}=\kappa \cosh \theta, \kappa_{n}=\kappa \sinh \theta, \tau_{g}=\tau+\frac{d \theta}{d s}$.

Timelike surface. The relation between Darboux frame and Frenet frame for a spacelike curve with a spacelike normal lying on a timelike surface is given by (4), where $\langle T, T\rangle=1,\langle Z, Z\rangle=1,\langle Y, Y\rangle=-1$. After using equations (1) and (4) we obtain in this case that

$$
\left|\begin{array}{c}
T^{\prime} \\
Y^{\prime} \\
Z^{\prime}
\end{array}\right|=\left|\begin{array}{ccc}
0 & \kappa_{n} & \kappa_{g} \\
\kappa_{n} & 0 & \tau_{g} \\
-\kappa_{g} & \tau_{g} & 0
\end{array}\right|\left|\begin{array}{c}
T \\
Y \\
Z
\end{array}\right|,
$$

where $\langle T, T\rangle=\langle Z, Z\rangle=1,\langle Y, Y\rangle=-1, T \times Z=-Y, Z \times Y=T, Y \times T=Z$, and $\kappa_{g}=\kappa \cosh \theta, \kappa_{n}=\kappa \sinh \theta, \tau_{g}=\tau+\frac{d \theta}{d s}$.

The relation between Darboux frame and Frenet frame for a spacelike curve with a timelike normal lying on a timelike surface is given by

$$
\left|\begin{array}{c}
T \\
Y \\
Z
\end{array}\right|=\left|\begin{array}{ccc}
1 & 0 & 0 \\
0 & \cosh (\theta) & \sinh (\theta) \\
0 & \sinh (\theta) & \cosh (\theta)
\end{array}\right|\left|\begin{array}{c}
T \\
N \\
B
\end{array}\right|,
$$


where $\langle T, T\rangle=1,\langle Z, Z\rangle=1,\langle Y, Y\rangle=-1$. After using equations (1) and (7) we can write that

$$
\left|\begin{array}{c}
T^{\prime} \\
Y^{\prime} \\
Z^{\prime}
\end{array}\right|=\left|\begin{array}{ccc}
0 & \kappa_{g} & \kappa_{n} \\
\kappa_{g} & 0 & \tau_{g} \\
-\kappa_{n} & \tau_{g} & 0
\end{array}\right|\left|\begin{array}{c}
T \\
Y \\
Z
\end{array}\right|,
$$

where $\langle T, T\rangle=\langle Z, Z\rangle=1,\langle Y, Y\rangle=-1, T \times Z=-Y, Z \times Y=T, Y \times T=Z$, and $\kappa_{g}=\kappa \cosh \theta, \kappa_{n}=-\kappa \sinh \theta, \tau_{g}=\tau+\frac{d \theta}{d s}$.

In the following let the vectors $V_{k}, K \in\{1,2,3\}$, be given as $V_{1}=T, V_{2}=Y$, $V_{3}=Z$, and let a fixed direction vector $U$ define the axis for the helix.

Definition 2. A spacelike curve $\gamma(s)$ on a spacelike or a timelike surface is said to be a $K$-type spacelike slant helix if there exists a non-zero fixed direction vector $U$ such that $\left\langle V_{K}, U\right\rangle=C$, where $C$ is a real constant and $K \in\{1,2,3\}$. But if the normal for a $K$-type spacelike slant helix is zero, then it is also known as a $K$-type pseudo null slant helix.

Note 1. The Darboux frame vector field $V_{K}$ cannot be constant because if it is constant, then the relation $\left\langle V_{k}, U\right\rangle=C$ is always satisfied, and there is nothing left to calculate.

\section{A 1-type spacelike slant helix with a spacelike or a timelike normal on a spacelike or a timelike surface}

Theorem 1. A spacelike curve $\gamma$ having a spacelike principal normal on a spacelike surface $M \subset E_{1}^{3}$ with an axis $U$ not orthogonal to $T$ is a 1-type spacelike slant helix if and only if it satisfies the condition

$$
\kappa_{g} e^{-\int \frac{\kappa_{g} \tau_{g}}{\kappa_{n}} d s} \int \kappa_{g} e^{\int \frac{\kappa_{g} \tau_{g}}{\kappa_{n}} d s} d s-\kappa_{n} e^{-\int \frac{\kappa_{n} \tau_{g}}{\kappa_{g}} d s} \int \kappa_{n} e^{\int \frac{\kappa_{n} \tau_{g}}{\kappa_{g}} d s} d s=0,
$$

where $\kappa_{n} \neq 0, \kappa_{g} \neq 0$, and $\tau_{g} \neq 0$.

Proof. Let $\gamma$ be a 1-type spacelike slant helix with a spacelike normal on a spacelike surface $M$. Then by Definition 2 there exists a constant direction vector $U$ such that

$$
\left\langle V_{1}, U\right\rangle=\langle T, U\rangle=C, \quad C \in \mathbb{R}
$$

Since the axis is not orthogonal to $T$, we have $C \neq 0$. Now with respect to Darboux frame $\{T, Y, Z\}$ the fixed direction vector $U$ can be expanded as

$$
U=C T+f Y+g Z,
$$

where $C$ is a constant and $f, g$ are functions of the parameter $s$. Differentiating equation (10), using equation (3), and comparing both sides of the 
equations, we get

$$
\left\{\begin{array}{l}
-\kappa_{g} f+\kappa_{n} g=0 \\
f^{\prime}+C \kappa_{g}+\tau_{g} g=0 \\
g^{\prime}+C \kappa_{n}+\tau_{g} f=0
\end{array}\right.
$$

Thus from $2^{\text {nd }}$ and $3^{\text {rd }}$ part of equation (11), we obtain the following set of solutions of equations:

$$
\left\{\begin{array}{l}
f=-C e^{-\int \frac{\kappa_{g} \tau_{g}}{\kappa_{n}} d s} \int \kappa_{g} e^{\int \frac{\kappa_{g} \tau_{g}}{\kappa_{n}} d s} d s, \\
g=-C e^{-\int \frac{\kappa_{n} \tau_{g}}{\kappa_{g}} d s} \int \kappa_{n} e^{\int \frac{\kappa_{n} \tau_{g}}{\kappa_{g}} d s} d s .
\end{array}\right.
$$

Substituting equation (12) into the first part of the equation (11), we get (9), where $\kappa_{n} \neq 0, \kappa_{g} \neq 0$ and $\tau_{g} \neq 0$.

Conversely, assume that $\gamma$ is a spacelike curve with a spacelike normal lying on a spacelike surface $M$. Let there exists a vector $U$ defined by

$U=C T-C e^{-\int \frac{\kappa_{g} \tau_{g}}{\kappa_{n}} d s}\left(\int \kappa_{g} e^{\int \frac{\kappa_{g} \tau_{g}}{\kappa_{n}} d s} d s\right) Y-C e^{-\int \frac{\kappa_{n} \tau_{g}}{\kappa_{g}} d s}\left(\int \kappa_{n} e^{\int \frac{\kappa_{n} \tau_{g}}{\kappa_{g}} d s} d s\right) Z$

and satisfying condition (9), where $\kappa_{n} \neq 0, \kappa_{g} \neq 0, \tau_{g} \neq 0$, and $C \in \mathbb{R}$. Now, differentiating the vector $U$ and using the given condition, we obtain $U^{\prime}=0$. Hence $U$ is a fixed direction vector such that, $\langle T, U\rangle=C$. Thus $\gamma$ is a 1-type spacelike slant helix with an axis $U$, not orthogonal to $T$.

Corollary 1. If $\gamma$ is a 1-type spacelike slant helix with a spacelike normal on a spacelike surface, then the axis for $\gamma$ is given by (13) with $\kappa_{n} \neq 0$, $\kappa_{g} \neq 0, \tau_{g} \neq 0$, and $C \in \mathbb{R}$.

Proof. Substitution of $f$ and $g$ from equation (12) into equation (10) yields the equation (13).

Corollary 2. A 1-type spacelike slant helix with a spacelike normal whose axis is not orthogonal to $T$ curvatures $\kappa_{g}, \kappa_{n}, \tau_{g}$ is neither 2-type nor 3-type spacelike slant helix with a spacelike normal.

Proof. From equations (13) and (2) we obtain the following set of equations:

$$
\left\{\begin{array}{l}
\langle T, U\rangle=C, \\
\langle Y, U\rangle=-C e^{-\int \frac{\kappa_{g} \tau_{g}}{\kappa_{n}} d s} \int \kappa_{g} e^{\int \frac{\kappa_{g} \tau_{g}}{\kappa_{n}} d s} d s, \\
\langle Z, U\rangle=C e^{-\int \frac{\kappa_{n} \tau_{g}}{\kappa_{g}} d s} \int \kappa_{n} e^{\int \frac{\kappa_{n} \tau_{g}}{\kappa_{g}} d s} d s .
\end{array}\right.
$$

Since $\kappa_{n} \neq 0, \kappa_{g} \neq 0$, and $\tau_{g} \neq 0$, from these equations we conclude that both $\langle Y, U\rangle$ and $\langle Z, U\rangle$ are not constant. Thus from Definition 2 we can conclude that a 1-type spacelike slant helix with an axis $U$ not orthogonal to $T$ is neither 2-type nor 3-type spacelike slant helix with an axis $U$. 
Theorem 2. If $\gamma$ is a spacelike curve with a spacelike normal on a spacelike surface $M \subset E_{1}^{3}$, then $\gamma$ is a 1-type spacelike slant helix whose axis is orthogonal to $T$ if and only if it satisfies the relation

$$
-C_{1} \kappa_{g} e^{-\int \frac{\kappa_{g} \tau g}{\kappa_{n}} d s}+C_{2} \kappa_{n} e^{-\int \frac{\kappa_{n} \tau_{g}}{\kappa_{g}} d s}=0,
$$

where $\kappa_{n} \neq 0, \kappa_{g} \neq 0, \tau_{g} \neq 0$, and $C_{1}, C_{2} \in \mathbb{R}$.

Proof. Let $\gamma$ be a 1-type spacelike slant helix with a spacelike normal on a spacelike surface $M$. Since the axis $U$ of $\gamma$ is orthogonal to $T$, by Definition 2 we have

$$
\left\langle V_{1}, U\right\rangle=\langle T, U\rangle=0 .
$$

Now with respect to Darboux frame $\{T, Y, Z\}$ the fixed direction vector $U$ can be expanded as

$$
U=f Y+g Z,
$$

where $f, g$ are functions of the parameter $s$. Differentiating equation (15), using equation (3), and comparing both sides of the equations, we get the system of equations

$$
\left\{\begin{array}{l}
-\kappa_{g} f+\kappa_{n} g=0, \\
f^{\prime}+\tau_{g} g=0, \\
g^{\prime}+\tau_{g} f=0 .
\end{array}\right.
$$

Thus from $2^{\text {nd }}$ and $3^{\text {rd }}$ part of equations (16) we have

$$
\left\{\begin{array}{l}
f=C_{1} e^{-\int \frac{\kappa_{g} \tau g}{\kappa_{n}} d s}, \\
g=C_{2} e^{-\int \frac{\kappa_{n} \tau g}{\kappa_{g}} d s} .
\end{array}\right.
$$

Substituting equation (17) into the first part of equation (16), we get (14).

Conversely, assume that $\gamma$ is a spacelike curve with a spacelike normal lying on a spacelike surface $M$. Assume there exists a vector

$$
U=C_{1} e^{-\int \frac{\kappa_{g} \tau_{g}}{\kappa_{n}} d s} Y+C_{2} e^{-\int \frac{\kappa_{n} \tau_{g}}{\kappa_{g}} d s} Z
$$

where $C_{1}, C_{2} \in \mathbb{R}$, and vector $U$ satisfies the condition

$$
-C_{1} \kappa_{g} e^{-\int \frac{\kappa_{g} \tau_{g}}{\kappa_{n}} d s}+C_{2} \kappa_{n} e^{-\int \frac{\kappa_{n} \tau_{g}}{\kappa_{g}} d s}=0,
$$

where $\kappa_{n} \neq 0, \kappa_{g} \neq 0, \tau_{g} \neq 0$. Now, differentiating vector $U$ and using the above condition, we obtain $U^{\prime}=0$. Hence $U$ is a fixed direction vector such that $\langle T, U\rangle=0$. Thus $\gamma$ is a 1-type spacelike slant helix whose axis $U$ is orthogonal to $T$.

Corollary 3. The axis for 1-type spacelike slant helix with a spacelike normal, whose axis is orthogonal to $T$, is given by

$$
U=-C_{1} e^{-\int \frac{\kappa_{g} \tau_{g}}{\kappa_{n}} d s} Y+C_{2} e^{-\int \frac{\kappa_{n} \tau_{g}}{\kappa_{g}} d s} Z .
$$

where $\kappa_{n} \neq 0, \kappa_{g} \neq 0, \tau_{g} \neq 0$, and $C_{1}, C_{2} \in \mathbb{R}$. 
Proof. Substituting $f, g$ from equation (17) into equation (15), we get the equation (18).

Theorem 3. If $\gamma$ be a spacelike curve with a spacelike normal and zero geodesic torsion $\left(\tau_{g}=0\right)$ lying on a spacelike surface $M \subset E_{1}^{3}$, then $\gamma$ is a 1-type spacelike slant helix if and only if non-zero curvature functions $\kappa_{n}$ and $\kappa_{g}$ satisfy the relation

$$
\kappa_{g} \int \kappa_{g} d s-\kappa_{n} \int \kappa_{n} d s=0 .
$$

Proof. Let $\gamma$ be a 1-type spacelike slant helix with a spacelike normal and zero geodesic torsion $\left(\tau_{g}=0\right)$ lying on a spacelike surface $M \subset E_{1}^{3}$. Then by Definition 2 there exists a constant direction vector $U$ satisfying the relation

$$
\left\langle V_{1}, U\right\rangle=\langle T, U\rangle=C, \quad C \in \mathbb{R} .
$$

Now with respect to Darboux frame $\{T, Y, Z\}$ the fixed direction vector $U$ can be decomposed as

$$
U=C T+f Y+g Z,
$$

where $C$ is a constant and $f, g$ are functions of the parameter $s$. Now, differentiating equation (20) and using equation (3), we get the set of equations

$$
\left\{\begin{array}{l}
-\kappa_{g} f+\kappa_{n} g=0, \\
f^{\prime}+C \kappa_{g}=0, \\
g^{\prime}+C \kappa_{n}=0 .
\end{array}\right.
$$

Thus from $2^{\text {nd }}$ and $3^{\text {rd }}$ part of equation (21) we have

$$
\left\{\begin{array}{l}
f=-C \int \kappa_{g} d s \\
g=-C \int \kappa_{n} d s
\end{array}\right.
$$

Substituting the equations (22) into the first part of equation (21), we get (19).

To prove the converse part, assume that $\gamma$ is a spacelike curve with a spacelike normal and $\tau_{g}=0$ lying on $M$. Let there exists a vector

$$
U=C T-C\left(\int \kappa_{g} d s\right) Y-C\left(\int \kappa_{n} d s\right) Z,
$$

satisfying the condition (19). Now, differentiating the vector $U$ and using given equation, we get $U^{\prime}=0$. Hence $U$ is a fixed direction vector. Thus $\gamma$ is a 1-type spacelike slant helix with $\tau_{g}=0$.

Corollary 4. The axis for 1-type spacelike slant helix with a spacelike normal and zero geodesic torsion $\tau_{g}=0$ is spanned by (23), where $\kappa_{n} \neq 0$, $\kappa_{g} \neq 0$, and $C \in \mathbb{R}$. 
Proof. Substituting $f, g$ from the set of solutions of equations (22) into equation (20), we get equation (23).

Note 2. (i) If $\gamma$ is a spacelike curve with a timelike principal normal on a timelike surface, then $\gamma$ is a 1-type spacelike slant helix with the axis not orthogonal to $T$ if and only if it satisfies the condition given in equation (9) and the axis for curve $\gamma$ is given by equation (13).

(ii) If $\gamma$ is a spacelike curve with a timelike normal on a timelike surface, then $\gamma$ would be a 1-type spacelike slant helix with the axis orthogonal to $T$ if and only if it satisfies the condition given in equation (14) and axis for curve $\gamma$ is given by (18).

Note 3. The results for axis orthogonal to $T$ are the same for 1-type spacelike slant helix with a timelike normal in a timelike surface are the same as that of 1-type spacelike slant helix with a spacelike normal on a spacelike surface.

\section{A 2-type spacelike slant helix with a spacelike or a timelike normal on a spacelike or a timelike surface}

Theorem 4. A spacelike curve $\gamma$ having a spacelike principal normal on a spacelike surface $M \subset E_{1}^{3}$ with the axis $U$ not orthogonal to $T$ is a 2-type spacelike slant helix if and only if it satisfies the condition

$$
\kappa_{g} e^{\int \frac{\kappa_{g} \kappa_{n}}{\tau g} d s} \int \kappa_{g} e^{-\int \frac{\kappa_{g} \kappa_{n}}{\tau_{g}} d s} d s-\tau_{g} e^{\int \frac{\kappa_{n} \tau_{g}}{\kappa_{g}} d s} \int \tau_{g} e^{-\int \frac{\kappa_{n} \tau_{g}}{\kappa_{g}} d s} d s=0,
$$

where $\kappa_{n} \neq 0, \kappa_{g} \neq 0$, and $\tau_{g} \neq 0$.

Proof. Let $\gamma$ be a 2-type spacelike slant helix with a spacelike normal on a spacelike surface $M$. By Definition 2, there exists a constant direction vector $U$ which satisfies the relation

$$
\left\langle V_{2}, U\right\rangle=\langle Y, U\rangle=C, \quad C \in \mathbb{R} .
$$

Since the axis is not orthogonal to $Y$, we have $C \neq 0$. Now with respect to Darboux frame $\{T, Y, Z\}$ the fixed direction vector $U$ can be expanded as

$$
U=f T+C Y+g Z,
$$

where $C$ is a constant and $f, g$ are functions of the parameter $s$. Now, differentiating equation (25) and using equation (3), we get

$$
\left\{\begin{array}{l}
\kappa_{g} f+\tau_{g} g=0 \\
f^{\prime}-C \kappa_{g}+\kappa_{n} g=0 \\
g^{\prime}+C \tau_{g}+\kappa_{n} f=0
\end{array}\right.
$$


Thus from the $2^{\text {nd }}$ and $3^{\text {rd }}$ part of equation (26) we obtain the following set of solutions:

$$
\left\{\begin{array}{l}
f=C e^{\int \frac{\kappa_{g} \kappa_{n}}{\tau_{g}} d s} \int \kappa_{g} e^{-\int \frac{\kappa_{g} \kappa_{n}}{\tau_{g}} d s} d s, \\
g=-C e^{\int \frac{\kappa_{n} \tau_{g}}{\kappa_{g}} d s} \int \tau_{g} e^{-\int \frac{\kappa_{n} \tau_{g}}{\kappa_{g}} d s} d s .
\end{array}\right.
$$

Substituting equation (27) into the first part of equation (26), we get (24).

Conversely, assume that $\gamma$ is a spacelike curve with a spacelike normal lying on a spacelike surface $M$. Suppose there exists a vector

$$
U=C e^{\int \frac{\kappa_{g} \kappa_{n}}{\tau_{g}} d s} \int \kappa_{g} e^{-\int \frac{\kappa_{g} \kappa_{n}}{\tau_{g}} d s} d s T+C Y-C e^{\int \frac{\kappa_{n} \tau_{g}}{\kappa_{g}} d s} \int \tau_{g} e^{-\int \frac{\kappa_{n} \tau_{g}}{\kappa_{g}} d s} d s Z,
$$

such that (24) holds. where $\kappa_{n} \neq 0, \kappa_{g} \neq 0$, and $\tau_{g} \neq 0$. Now, differentiating the vector $U$ and using the given condition, we obtain $U^{\prime}=0$. Hence $U$ is a fixed direction vector such that $\langle Y, U\rangle=C$. Thus $\gamma$ is a 2-type spacelike slant helix with an axis $U$ not orthogonal to $Y$.

Corollary 5. If $\gamma$ is a 2-type spacelike slant helix with a spacelike normal on a spacelike surface, then the axis for $\gamma$ is given by (28), where $\kappa_{n} \neq 0$, $\kappa_{g} \neq 0$, and $\tau_{g} \neq 0$.

Proof. Substituting solutions $f, g$ from (27) into equation (25), we get (28).

Corollary 6. A 2-type spacelike slant helix with spacelike normal whose axis is not orthogonal to $Y$ curvatures $\kappa_{g}, \kappa_{n}, \tau_{g}$ is neither 1-type nor 3-type spacelike slant helix with spacelike normal.

Proof. From equation (28) and the sub-case (2) we obtain the set of equations

$$
\left\{\begin{array}{l}
\langle T, U\rangle=C e^{\int \frac{\kappa_{g} \kappa_{n}}{\tau_{g}} d s} \int \kappa_{g} e^{-\int \frac{\kappa_{g} \kappa n}{\tau_{g}} d s} d s, \\
\langle Y, U\rangle=C, \\
\langle Z, U\rangle=C e^{\int \frac{\kappa_{n} \tau_{g}}{\kappa g} d s} \int \tau_{g} e^{-\int \frac{\kappa_{n} \tau_{g}}{\kappa_{g}} d s} d s .
\end{array}\right.
$$

Since $\kappa_{n} \neq 0, \kappa_{g} \neq 0$, and $\tau_{g} \neq 0$, from equation (29) we conclude that $\langle T, U\rangle$, $\langle Z, U\rangle$ both are not constants. Thus from Definition 2 we conclude that a 2-type spacelike slant helix with an axis $U$ not orthogonal to $Y$ is neither 1-type nor 3-type spacelike slant helix with axis $U$.

Theorem 5. If $\gamma$ is a spacelike curve with a spacelike normal on a spacelike surface $M \subset E_{1}^{3}$, then $\gamma$ is a 2-type slant helix whose axis is orthogonal to $Y$ if and only if it satisfies the relation

$$
C_{1} \kappa_{g} e^{\int \frac{\kappa_{g} \kappa_{n}}{\tau_{g}}} d s+C_{2} \tau_{g} e^{\int \frac{\kappa_{n} \tau_{g}}{\kappa_{g}} d s}=0,
$$

where $\kappa_{n} \neq 0, \kappa_{g} \neq 0, \tau_{g} \neq 0$. 
Proof. Let $\gamma$ be a 2-type spacelike slant helix with a spacelike normal on a spacelike surface $M$ and let the axis of $\gamma$ be orthogonal to $Y$. Then by Definition 2 we get

$$
\left\langle V_{2}, U\right\rangle=\langle Y, U\rangle=0 .
$$

Now with respect to Darboux frame $\{T, Y, Z\}$ the fixed direction vector $U$ can be decomposed as

$$
U=f T+g Z,
$$

where $f, g$ are functions of a parameter $s$. Differentiating equation (31) and using equation (3), we get the set of equations

$$
\left\{\begin{array}{l}
\kappa_{g} f+\tau_{g} g=0, \\
f^{\prime}+\kappa_{n} g=0, \\
g^{\prime}+\kappa_{n} f=0 .
\end{array}\right.
$$

Thus from the $2^{\text {nd }}$ and $3^{\text {rd }}$ part of equation (32) we have

$$
\left\{\begin{array}{l}
f=C_{1} e^{\int \frac{\kappa_{g} \kappa n}{\tau_{g}}} d s, \\
g=C_{2} e^{\int \frac{\kappa n \tau_{g}}{\kappa g} d s} .
\end{array}\right.
$$

Substituting equation (33) in the first equation of (32), we get (30).

Conversely, suppose that $\gamma$ is a spacelike curve with a spacelike normal lying on a spacelike surface $M$. Let there exists a vector

$$
U=C_{1} e^{\int \frac{\kappa g \kappa n}{\tau_{g}}} d s T+C_{2} e^{\int \frac{\kappa n \tau g}{\kappa g} d s} Z,
$$

with $C_{1}, C_{2} \in \mathbb{R}$, and suppose that (30) holds, where $\kappa_{n} \neq 0, \kappa_{g} \neq 0, \tau_{g} \neq 0$. Now, differentiating the vector $U$ and using the above condition, we obtain that $U^{\prime}=0$. Hence $U$ is a fixed direction vector such that, $\langle Y, U\rangle=0$. Thus $\gamma$ is a 2-type spacelike slant helix whose axis $U$ is orthogonal to $Y$.

Corollary 7. The axis for a 2-type spacelike slant helix with a spacelike normal whose axis is orthogonal to $Y$ is given by

$$
U=C_{1} e^{\int \frac{\kappa_{g} \kappa_{n}}{\tau_{g}} d s} T+C_{2} e^{\int \frac{\kappa_{n} \tau_{g}}{\kappa_{g}} d s},
$$

where $\kappa_{n} \neq 0, \kappa_{g} \neq 0, \tau_{g} \neq 0$, and $C_{1}, C_{2} \in \mathbb{R}$.

Proof. Substituting $f, g$ from the set of equations (33) into equation (31), we get the equation (35).

Theorem 6. If $\gamma$ is a spacelike curve with a spacelike normal and zero geodesic torsion $\tau_{g}$ lying on $M \subset E_{1}^{3}$, then $\gamma$ is a 2-type spacelike slant helix if and only if the ratio of curvature functions $\kappa_{n}$ and $\kappa_{g}$ is constant. 
Proof. Let $\gamma$ be a 2-type spacelike slant helix with a spacelike normal and zero geodesic torsion $\tau_{g}$ lying on a surface $M$. Then by Definition 2 there exists a constant direction vector $U$ satisfying the relation

$$
\left\langle V_{2}, U\right\rangle=\langle Y, U\rangle=C, \quad C \in \mathbb{R} .
$$

Now with respect to Darboux frame $\{T, Y, Z\}$ the fixed direction vector $U$ can be decomposed as

$$
U=f T+C Y+g Z,
$$

where $C$ is a constant and $f, g$ are functions of the parameter $s$. Now, differentiating equation (36) and using equation (3), we get the system of equations

$$
\left\{\begin{array}{l}
\kappa_{g} f=0, \\
f^{\prime}-C \kappa_{g}+\kappa_{n} g=0, \\
g^{\prime}+\kappa_{n} f=0
\end{array}\right.
$$

After solving these equations we get $f=0, g=A=$ constant. Therefore,

$$
\frac{\kappa_{n}}{\kappa_{g}}=\frac{C}{A}=\text { constant. }
$$

Conversely, let $\gamma$ be a spacelike curve with a spacelike normal and $\tau_{g}=0$ lying on $M \subset E_{1}^{3}$. Let there exist a vector $U=C Y+A_{1} Z$, where $\kappa_{n} \neq 0$, $\kappa_{g} \neq 0$ and $C \frac{\kappa_{g}}{\kappa_{n}}=A_{1} \in \mathbb{R}$. Now, differentiating the vector $U$ we obtain $U^{\prime}=$ 0 . Hence $U$ is a fixed direction vector such that $\langle U, Y\rangle=C$ and $\langle U, Z\rangle=A_{1}$. Thus $\gamma$ is a 2-type and 3-type spacelike slant helix.

Corollary 8. The axis for a 2-type spacelike slant helix with a spacelike normal and zero geodesic torsion $\tau_{g}$ lying on $M \subset E_{1}^{3}$, is spanned by

$$
U=C Y+A_{1} Z,
$$

where $\kappa_{n} \neq 0, \kappa_{g} \neq 0$, and $C \frac{\kappa_{g}}{\kappa_{n}}=A_{1} \in \mathbb{R}$.

Proof. Substituting $f$ and $g$ from the set of equations (37) into equation (36), we get the equation (38).

Note 4. If $\gamma$ is a spacelike curve with a timelike normal on a timelike surface $M \subset E_{1}^{3}$, then $\gamma$ is a 2-type spacelike slant helix on a timelike surface with the axis not orthogonal to $Y$ if and only if it satisfies the condition

$$
\kappa_{g} e^{-\int \frac{\kappa_{g} \kappa_{n}}{\tau g} d s} \int \kappa_{g} e^{\int \frac{\kappa_{g} \kappa_{n}}{\tau_{g}} d s} d s+\tau_{g} e^{\int \frac{\kappa_{n} \tau_{g}}{\kappa_{g}} d s} \int \tau_{g} e^{-\int \frac{\kappa_{n} \tau_{g}}{\kappa_{g}} d s} d s=0,
$$

where $\kappa_{n} \neq 0, \kappa_{g} \neq 0$, and $\tau_{g} \neq 0$.

Note 5. The axis for a 2-type spacelike slant helix with a timelike normal on a timelike surface $M \subset E_{1}^{3}$ not orthogonal to $Y$ is given by the equation $U=-C e^{-\int \frac{\kappa_{g} \kappa_{n}}{\tau_{g}} d s} \int \kappa_{g} e^{\int \frac{\kappa_{g} \kappa_{n}}{\tau_{g}} d s} d s T+C Y-C e^{\int \frac{\kappa_{n} \tau_{g}}{\kappa_{g}} d s} \int \tau_{g} e^{-\int \frac{\kappa_{n} \tau_{g}}{\kappa_{g}} d s} d s Z$, 
where $\kappa_{n} \neq 0, \kappa_{g} \neq 0, \tau_{g} \neq 0$, and $C \in \mathbb{R}$.

Note 6. If $\gamma$ is a spacelike curve with a timelike normal on a timelike surface $M \subset E_{1}^{3}$, then $\gamma$ is a 2-type spacelike slant helix on a timelike surface with the axis orthogonal to $Y$ if and only if it satisfies the relation

$$
C_{1} \kappa_{g} e^{-\int \frac{\kappa_{g} \kappa_{n}}{\tau_{g}} d s}+C_{2} \tau_{g} e^{\int \frac{\kappa_{n} \tau_{g}}{\kappa_{g}} d s}=0
$$

where $\kappa_{n} \neq 0, \kappa_{g} \neq 0, \tau_{g} \neq 0$, and $C_{1}, C_{2} \in \mathbb{R}$.

Note 7. The axis for a 2-type spacelike slant helix with a timelike normal on a timelike surface $M \subset E_{1}^{3}$, whose axis is orthogonal to $Y$, is spanned by

$$
C_{1} e^{-\int \frac{\kappa g \kappa n}{\tau_{g}} d s} T+C_{2} e^{\int \frac{\kappa n \tau g}{\kappa g} d s} Z,
$$

where $\kappa_{n} \neq 0, \kappa_{g} \neq 0, \tau_{g} \neq 0$, and $C_{1}, C_{2} \in \mathbb{R}$.

Note 8. For $\kappa_{n} \neq 0, \kappa_{g} \neq 0$, and $\tau_{g}=0$, the result concerning a 2-type spacelike slant helix with a timelike normal on a timelike surface is the same as that of a 2-type spacelike slant helix with a spacelike normal on a spacelike surface.

\section{A 3-type spacelike slant helix with a spacelike or a timelike normal on a spacelike or a timelike surface}

Theorems and corollaries for a 3-type spacelike slant helix with non-null normal can be proved in a similar way as for 1-type, 2-type spacelike slant helices in Sections 3 and 4 and their subsections, respectively.

Theorem 7. A spacelike curve $\gamma$ having a spacelike principal normal on a spacelike surface $M \subset E_{1}^{3}$ with the axis $U$ not orthogonal to $Z$ is a 3-type spacelike slant helix if and only if it satisfies the condition

$$
\kappa_{n} e^{-\int \frac{\kappa_{g} \kappa_{n}}{\tau_{g}} d s} \int \kappa_{n} e^{\int \frac{\kappa_{g} \kappa_{n}}{\tau_{g}} d s} d s+\tau_{g} e^{\int \frac{\kappa_{g} \tau_{g}}{\kappa_{n}} d s} \int \tau_{g} e^{-\int \frac{\kappa_{g} \tau_{g}}{\kappa_{n}} d s} d s=0,
$$

where $\kappa_{n} \neq 0, \kappa_{g} \neq 0, \tau_{g} \neq 0$, and $C \in \mathbb{R}$.

Corollary 9. If $\gamma$ is a 3-type spacelike slant helix with a spacelike normal on a spacelike surface, then the axis for $\gamma$ is given by

$$
U=-C e^{-\int \frac{\kappa_{g} \kappa_{n}}{\tau g} d s} \int \kappa_{n} e^{\int \frac{\kappa_{g} \kappa_{n}}{\tau_{g}} d s} d s T-C e^{\int \frac{\kappa_{g} \tau_{g}}{\kappa_{n}} d s} \int \tau_{g} e^{-\int \frac{\kappa_{g} \tau_{g}}{\kappa_{n}} d s} d s+C Z .
$$

where $\kappa_{n} \neq 0, \kappa_{g} \neq 0, \tau_{g} \neq 0$, and $C \in \mathbb{R}$.

Corollary 10. 3-type spacelike slant helices with spacelike normals whose axises are not orthogonal to $Z$ with non-zero constant curvatures $\kappa_{g}, \kappa_{n}, \tau_{g}$ are neither 1-type nor 2-type spacelike slant helices. 
Theorem 8. If $\gamma$ is a spacelike curve with a spacelike normal on a spacelike surface in $E_{1}^{3}$, then $\gamma$ is a 3-type spacelike slant helix whose axis is orthogonal to $Z$ if and only if it satisfies the relation

$$
C_{1} \kappa_{n} e^{-\int \frac{\kappa_{g} \kappa_{n}}{\tau_{g}} d s}+C_{2} \tau_{g} e^{\int \frac{\kappa_{g} \tau_{g}}{\kappa_{n}} d s}=0,
$$

where $\kappa_{n} \neq 0, \kappa_{g} \neq 0, \tau_{g} \neq 0$, and $C_{1}, C_{2} \in \mathbb{R}$.

Corollary 11. The axis for a 3-type spacelike slant helix with a spacelike normal whose axis is orthogonal to $Z$ is given by

$$
U=C_{1} e^{-\int \frac{\kappa g \kappa n}{\tau_{g}} d s} T+C_{2} e^{\int \frac{\kappa g \tau g}{\kappa n} d s} Y,
$$

where $\kappa_{n} \neq 0, \kappa_{g} \neq 0, \tau_{g} \neq 0$, and $C_{1}, C_{2} \in \mathbb{R}$.

Theorem 9. If $\gamma$ is a spacelike curve with a spacelike normal and zero geodesic torsion lying on a spacelike surface $M \subset E_{1}^{3}$, then $\gamma$ is a 3-type spacelike slant helix with $\kappa_{g} \neq 0, \kappa_{n} \neq 0$, and $\tau_{g}=0$ if and only if the ratio of $\kappa_{n}$ and $\kappa_{g}$ is constant.

Corollary 12. The axis for a 3-type spacelike slant helix with a spacelike normal and zero geodesic torsion $\tau_{g}$ lying on a spacelike surface $M \subset E_{1}^{3}$ is spanned by

$$
U=C \frac{\kappa_{n}}{\kappa_{g}}+C Z
$$

where $\kappa_{n} \neq 0, \tau_{g} \neq 0$, and $C \in \mathbb{R}$.

Note 9. If $\gamma$ is a spacelike curve with a timelike normal on a timelike surface $M \subset E_{1}^{3}$, then $\gamma$ is a 3-type spacelike slant helix on $M$ with the axis not orthogonal to $Z$ if and only if it satisfies the condition

$$
\kappa_{n} e^{\int \frac{\kappa_{g} \kappa_{n}}{\tau g} d s} \int \kappa_{n} e^{-\int \frac{\kappa_{g} \kappa_{n}}{\tau_{g}} d s} d s-\tau_{g} e^{\int \frac{\kappa_{g} \tau_{g}}{\kappa_{n}} d s} \int \tau_{g} e^{-\int \frac{\kappa_{g} \tau_{g}}{\kappa_{n}} d s} d s=0,
$$

where $\kappa_{n} \neq 0, \kappa_{g} \neq 0$, and $\tau_{g} \neq 0$.

Note 10. The axis for a 3-type spacelike slant helix with a timelike normal on a timelike surface $M \subset E_{1}^{3}$ not orthogonal to $Z$ is given by the equation

$$
U=C e^{\int \frac{\kappa_{g} \kappa_{n}}{\tau g} d s} \int \kappa_{n} e^{-\int \frac{\kappa_{g} \kappa_{n}}{\tau_{g}} d s} d s T-C e^{\int \frac{\kappa_{g} \tau_{g}}{\kappa_{n}} d s} \int \tau_{g} e^{-\int \frac{\kappa_{g} \tau_{g}}{\kappa_{n}} d s} d s+C Z,
$$

where $\kappa_{n} \neq 0, \kappa_{g} \neq 0, \tau_{g} \neq 0$, and $C \in \mathbb{R}$.

Note 11. If $\gamma$ is a spacelike curve with a timelike normal on a timelike surface $M \subset E_{1}^{3}$, then $\gamma$ is a 3 -type spacelike slant helix with the axis orthogonal to $Z$ if and only if

$$
C_{1} \kappa_{g} e^{\int \frac{\kappa_{g} \kappa_{n}}{\tau_{g}} d s}+C_{2} \tau_{g} e^{\int \frac{\kappa_{n} \tau_{g}}{\kappa_{g}} d s}=0,
$$

where $\kappa_{n} \neq 0, \kappa_{g} \neq 0, \tau_{g} \neq 0$, and $C_{1}, C_{2} \in \mathbb{R}$. 
Note 12. The axis for a 3-type spacelike slant helix with a timelike normal on a timelike surface $M \subset E_{1}^{3}$, whose axis is orthogonal to $Z$, is spanned by

$$
C_{1} e^{\int \frac{\kappa_{g} \kappa_{n}}{\tau_{g}} d s} T+C_{2} e^{\int \frac{\kappa_{n} \tau_{g}}{\kappa_{g}} d s} Z,
$$

where $\kappa_{n} \neq 0, \kappa_{g} \neq 0, \tau_{g} \neq 0$, and $C \in \mathbb{R}$.

Note 13. For $\kappa_{n} \neq 0, \kappa_{g} \neq 0$, and $\tau_{g}=0$, the result concerning a 3-type spacelike slant helix with a timelike normal on a timelike surface is the same as that of a 3-type spacelike slant helix with a spacelike normal on a spacelike surface.

\section{Examples}

Example 1. Let $M \subset E_{1}^{3}, M(s, t)=\left(-a \sinh \frac{s}{c},-\frac{b s}{c},-a \cosh \frac{s}{c}\right)+$ $t\left(-\sinh \frac{s}{c} \cosh \frac{2 b s}{c^{2}}+\frac{b}{c} \sinh \frac{2 b s}{c^{2}} \cosh \frac{s}{c}, \quad \frac{a}{c} \sinh \frac{2 b s}{c^{2}}, \quad-\cosh \frac{2 b s}{c^{2}} \cosh \frac{s}{c}+\right.$ $\left.\frac{b}{c} \sinh \frac{2 b s}{c^{2}} \sinh \frac{s}{c}\right)$ is a spacelike surface, and $\gamma(s)=\left(-a \sinh \frac{s}{c},-\frac{b s}{c}\right.$, $\left.-a \cosh \frac{s}{c}\right)$ with $\frac{b^{2}-a^{2}}{C^{2}}=1$ is a spacelike curve with a spacelike normal lying on a surface $M$. From Case 1 in Section 2, Frenet frame $\{T, N, B\}$ for the curve $\gamma$ is

$$
\begin{aligned}
T(s) & =\left(-\frac{a}{c} \cosh \frac{s}{c},-\frac{b}{c},-\frac{a}{c} \sinh \frac{s}{c}\right), \\
N(s) & =\left(-\sinh \frac{s}{c}, 0,-\cosh \frac{s}{c}\right), \\
B(s) & =\left(\frac{b}{c} \cosh \frac{s}{c}, \frac{a}{c}, \frac{b}{c} \sinh \frac{s}{c}\right) .
\end{aligned}
$$

Curvature functions for the curve are $\kappa(s)=\frac{a}{c^{2}}$ and $\tau(s)=\frac{-b}{c^{2}}$. Equation (2) provides the relation between Darboux frame and Frenet frame. Now, if we take $\theta(s)=\frac{2 b s}{c^{2}}$, then from (3) we get the following relations:

$$
\kappa_{g}=\kappa \cosh \frac{2 b s}{c^{2}}, \quad \kappa_{n}=-\kappa \sinh \frac{2 b s}{c^{2}}, \quad \tau_{g}=\frac{b}{c^{2}} .
$$

After substituting the values of $\kappa_{g}, \kappa_{n}$, and $\tau_{g}$ into condition given in Theorem 1, we find that $\gamma(s)$ is a 1-type spacelike slant helix with a spacelike normal. The curve $\gamma$ does not satisfy the condition given in Theorems 4 and 7 , so $\gamma$ is neither a 2-type nor a 3 -type slant helix. The Darboux frame vectors for $\gamma(s)$ are obtained from equation (2) and are given by

$$
\begin{aligned}
Y= & \left(-\sinh \frac{s}{c} \cosh \frac{2 b s}{c^{2}}+\frac{b}{c} \sinh \frac{2 b s}{c^{2}} \cosh \frac{s}{c}, \frac{a}{c} \sinh \frac{2 b s}{c^{2}},\right. \\
& \left.-\cosh \frac{2 b s}{c^{2}} \cosh \frac{s}{c}+\frac{b}{c} \sinh \frac{2 b s}{c^{2}} \sinh \frac{s}{c}\right),
\end{aligned}
$$




$$
\begin{aligned}
Z= & \left(-\sinh \frac{2 b s}{c^{2}} \sinh \frac{s}{c}+\frac{b}{c} \cosh \frac{2 b s}{c^{2}} \cosh \frac{s}{c}, \frac{a}{c} \cosh \frac{2 b s}{c^{2}},\right. \\
& \left.-\sinh \frac{2 b s}{c^{2}} \cosh \frac{s}{c}+\frac{b}{c} \cosh \frac{2 b s}{c^{2}} \sinh \frac{s}{c}\right) .
\end{aligned}
$$

From equation (12) we obtain the values for $f, g$ as follows:

$$
f=-C \frac{a}{b} \sinh \frac{2 b s}{c^{2}}, \quad g=C \frac{a}{b} \cosh \frac{2 b s}{c^{2}} .
$$

From Corollary 5 the axis for $\gamma(s)$ is not orthogonal to $T$ and is given by

$$
U=C T-C \frac{a}{b}\left(\sinh \frac{2 b s}{c^{2}}\right) Y+C \frac{a}{b}\left(\cosh \frac{2 b s}{c^{2}}\right) Z .
$$

From Theorem 4 and Theorem 7 we find that the curve $\gamma$ does not satisfy the provided conditions, hence $\gamma$ is neither a 2 -type nor a 3 -type spacelike slant helix.

Let $\mathrm{a}=3, \mathrm{~b}=5, \mathrm{c}=4,-\pi \leq s \leq \pi$, and $-100 \leq t \leq 100$ in this example. Then we get the following figures for a spacelike surface and a 1-type sacelike slant helix with a spacelike normal lying on this surface.

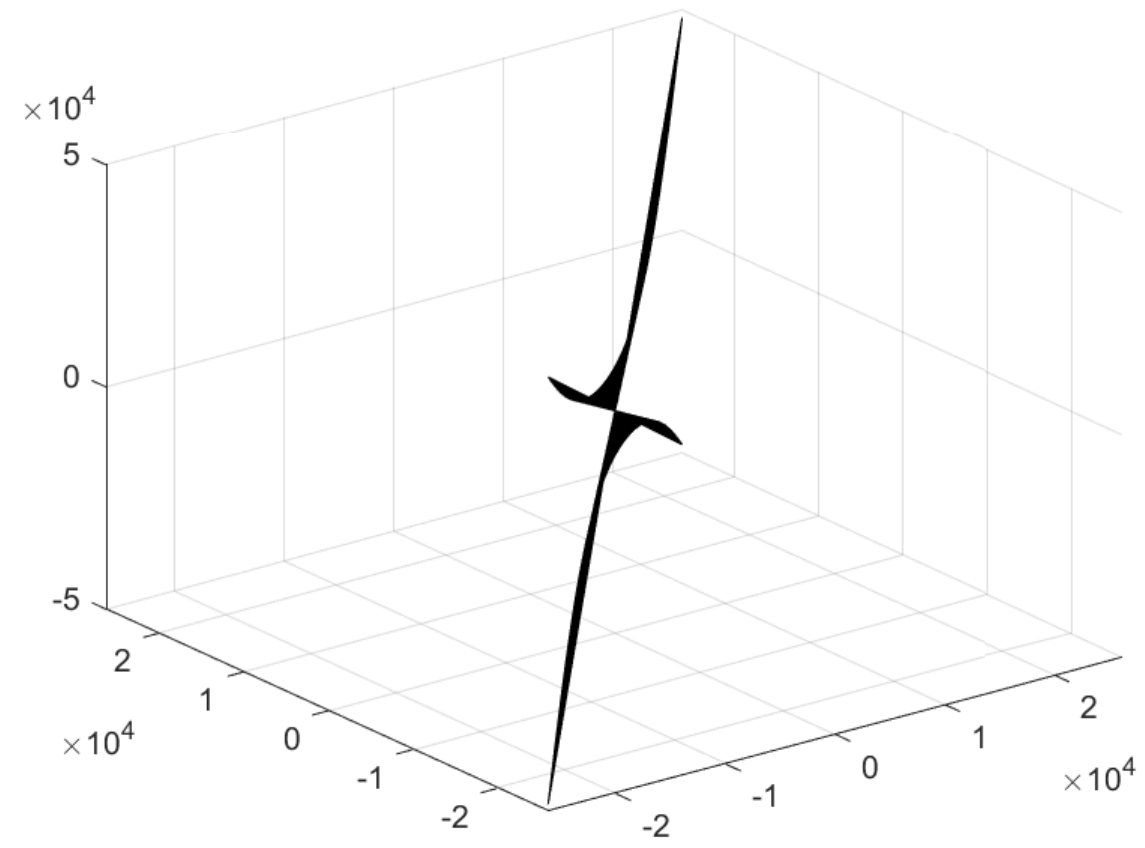

FIGURE 1. Spacelike surface $M(s, t)$. 


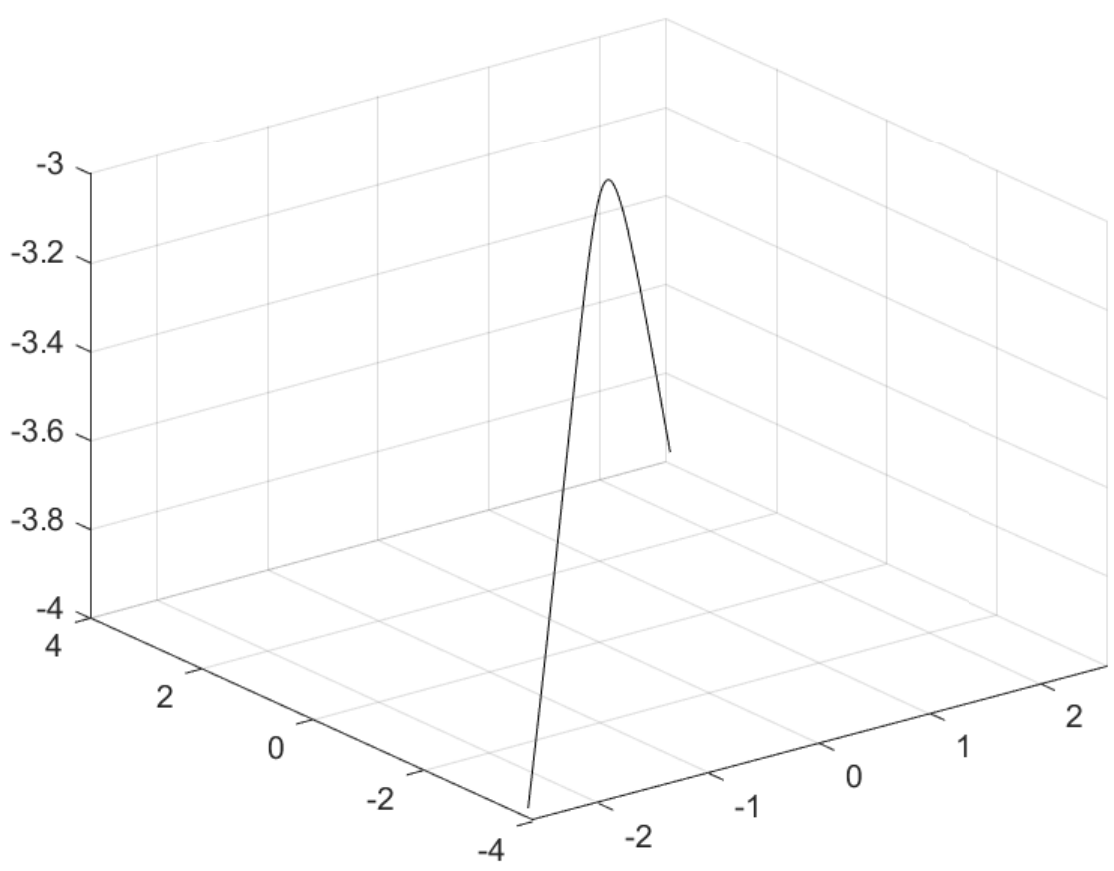

Figure 2. A 1-type spacelike slant helix on $M(s, t)$.

Example 2. Let $M \subset E_{1}^{3}, M(s, t)=\left(-a \sinh \frac{s}{c},-\frac{b s}{c},-a \cosh \frac{s}{c}\right)+$ $t\left(-\sinh \frac{s}{c} \cosh \frac{b s}{c^{2}}+\frac{b}{c} \sinh \frac{b s}{c^{2}} \cosh \frac{s}{c}, \frac{a}{c} \sinh \frac{b s}{c^{2}},-\cosh \frac{b s}{c^{2}} \cosh \frac{s}{c}+\frac{b}{c} \sinh \frac{b s}{c^{2}} \sinh \frac{s}{c}\right)$ be a spacelike surface, and $\gamma(s)=\left(-a \sinh \frac{s}{c},-\frac{b s}{c},-a \cosh \frac{s}{c}\right)$ with $\frac{b^{2}-a^{2}}{C^{2}}=1$ be a spacelike curve with zero geodesic torsion $\tau_{g}$ lying on a spacelike surface $M(s, t)$ in $E_{1}^{3}$. From Case 1 in Section 2, Frenet frame $\{T, N, B\}$ for the curve $\gamma$ is obtained as

$$
\begin{aligned}
T(s) & =\left(-\frac{a}{c} \cosh \frac{s}{c},-\frac{b}{c},-\frac{a}{c} \sinh \frac{s}{c}\right), \\
N(s) & =\left(-\sinh \frac{s}{c}, 0,-\cosh \frac{s}{c}\right), \\
B(s) & =\left(\frac{b}{c} \cosh \frac{s}{c}, \frac{a}{c}, \frac{b}{c} \sinh \frac{s}{c}\right) .
\end{aligned}
$$

Curvature functions for the curve is $\kappa(s)=\frac{a}{c^{2}}$ and $\tau(s)=\frac{-b}{c^{2}}$. Now, if we take $\theta(s)=\frac{b s}{c^{2}}$, then from 3 we get the relations

$$
\kappa_{g}=\kappa \cosh \frac{b s}{c^{2}}, \quad \kappa_{n}=-\kappa \sinh \frac{b s}{c^{2}}, \quad \tau_{g}=0 .
$$


Substitute the value of $\kappa_{g}$ and $\kappa_{n}$ in Theorem 3. We find that $\gamma(s)$ is a 1-type spacelike slant helix with a spacelike normal and zero geodesic torsion $\tau_{g}$ with $\kappa_{g} \neq 0, \kappa_{n} \neq 0$, and Darboux frame vector for $\gamma(s)$ from equation (2) is given by

$$
\begin{aligned}
Y= & \left(-\sinh \frac{s}{c} \cosh \frac{b s}{c^{2}}+\frac{b}{c} \sinh \frac{b s}{c^{2}} \cosh \frac{s}{c}, \frac{a}{c} \sinh \frac{b s}{c^{2}},\right. \\
& \left.-\cosh \frac{b s}{c^{2}} \cosh \frac{s}{c}+\frac{b}{c} \sinh \frac{b s}{c^{2}} \sinh \frac{s}{c}\right), \\
Z= & \left(-\sinh \frac{b s}{c^{2}} \sinh \frac{s}{c}+\frac{b}{c} \cosh \frac{b s}{c^{2}} \cosh \frac{s}{c}, \frac{a}{c} \cosh \frac{b s}{c^{2}},\right. \\
& \left.-\sinh \frac{b s}{c^{2}} \cosh \frac{s}{c}+\frac{b}{c} \cosh \frac{b s}{c^{2}} \sinh \frac{s}{c}\right) .
\end{aligned}
$$

From Corollary 4 the axis for $\gamma(s)$ whose geodesic torsion is equal to zero is given by

$$
U=C T-C \frac{a}{b}\left(\sinh \frac{b s}{c^{2}}\right) Y+C \frac{a}{b}\left(\cosh \frac{b s}{c^{2}}\right) Z .
$$

\section{A method for generating the $1^{\text {st }}$ axis for a $K$-type spacelike Darboux helix on a spacelike and a timelike surface}

Let $\gamma$ be a $K$-type spacelike slant helix with a non-null normal lying on a spacelike (timelike) surface. If there exists a constant direction vector $V$ not collinear to $U$ (the axis of slant helix) whose inner product with Darboux vector $(D)$ of curve $\gamma$ is constant, then the curve $\gamma$ is identified as a $K$-type spacelike Darboux helix and the Darboux vector $D$ is known as the first axis for the curve $\gamma$. Darboux vector for a spacelike curve with a spacelike (timelike) normal is given by [7]:

$$
D=\tau_{g} T-\kappa_{n} Y-\kappa_{g} Z .
$$

For a spacelike surface,

$$
T^{\prime}=D \wedge T, \quad Y^{\prime}=D \wedge Y, \quad Z^{\prime}=D \wedge Z .
$$

For a timelike surface,

$$
T^{\prime}=T \wedge D, \quad Y^{\prime}=Y \wedge D, \quad Z^{\prime}=Z \wedge D .
$$

Let $\gamma$ be a $K$-type spacelike slant helix with a spacelike normal lying on a spacelike surface and let $V$ be a fixed direction vector orthogonal to Darboux vector given by

$$
V=f T+g Y+h Z
$$


Differentiating the equation (40), we obtain the set of equations

$$
\left\{\begin{array}{l}
f^{\prime}-\kappa_{g} g+\kappa_{n} h=0, \\
g^{\prime}+\kappa_{g} f+\tau_{g} h=0, \\
h^{\prime}+\kappa_{n} f+\tau_{g} g=0 .
\end{array}\right.
$$

Since $V$ is a constant direction vector orthogonal to $D$, we have $\langle D, V\rangle=0$ and $\left\langle D^{\prime}, V\right\rangle=0$. Here $D^{\prime}=\tau_{g}^{\prime} T-\kappa_{n}^{\prime} Y-\kappa_{g}^{\prime} Z$ and, taking the inner product of $D$ and $D^{\prime}$ with the constant direction vector $U$, we obtain the set of equations

$$
\left\{\begin{array}{l}
\tau_{g} f-\kappa_{n} g+\kappa_{g} h=0, \\
\tau_{g}^{\prime} f-\kappa_{n}^{\prime} g+\kappa_{g}^{\prime} h=0 .
\end{array}\right.
$$

After solving equations (41) and (42) we get the following values:

$$
\begin{aligned}
& f=C_{1} e^{\frac{1}{2} \int \frac{\tau_{g}\left(\kappa_{n}^{2}-\kappa_{g}^{2}\right)^{\prime}-2 \tau_{g}^{\prime}\left(\kappa_{n}^{2}-\kappa_{g}^{2}\right)}{\kappa g \kappa_{n}^{\prime}-\kappa_{g}^{\prime} \kappa_{n}} d s} \\
& =C_{2} \frac{\kappa_{g} \kappa_{n}^{\prime}-\kappa_{g}^{\prime} \kappa_{n}}{\kappa_{g} \tau_{g}^{\prime}-\kappa_{g}^{\prime} \tau_{g}} e^{\frac{1}{2} \int \frac{\kappa_{n}\left(\kappa_{g}^{2}-\tau_{g}^{2}\right)^{\prime}-2 \kappa_{n}^{\prime}\left(\kappa_{g}^{2}-\tau_{g}^{2}\right)}{\kappa_{g} \tau_{g}^{\prime}-\tau_{g} \kappa_{g}^{\prime}} d s} \\
& =-C_{3} \frac{\kappa_{g} \kappa_{n}^{\prime}-\kappa_{g}^{\prime} \kappa_{n}}{\kappa_{n}^{\prime} \tau_{g}-\kappa_{n} \tau_{g}^{\prime}} e^{\frac{1}{2} \int \frac{\kappa_{g}\left(\kappa_{n}^{2}-\tau_{g}^{2}\right)^{\prime}-2 \kappa_{g}^{\prime}\left(\kappa_{n}^{2}+\tau_{g}^{2}\right)}{\kappa_{n}^{\prime} \tau_{g}-\tau_{g}^{\prime} \kappa_{n}} d s} . \\
& g=C_{2} e^{\frac{1}{2} \int \frac{\kappa_{n}\left(\kappa_{g}^{2}-\tau_{g}^{2}\right)^{\prime}-2 \kappa_{n}^{\prime}\left(\kappa_{g}^{2}-\tau_{2}^{2}\right)}{\kappa_{g} \tau_{g}^{\prime}-\tau_{g} \kappa_{g}^{\prime}} d s} \\
& =C_{1} \frac{\tau_{g}^{\prime} \kappa_{g}-\kappa_{g}^{\prime} \tau_{g}}{\kappa_{g} \kappa_{n}^{\prime}-\kappa_{g}^{\prime} \kappa_{n}} e^{\frac{1}{2} \int \frac{\tau_{g}\left(\kappa_{n}^{2}-\kappa_{g}^{2}\right)^{\prime}-2 \tau_{g}^{\prime}\left(\kappa_{n}^{2}-\kappa_{g}^{2}\right)}{\kappa_{g} \kappa_{n}^{\prime}-\kappa_{g}^{\prime} \kappa_{n}} d s} \\
& =C_{3} \frac{\kappa_{g} \tau_{g}^{\prime}-\tau_{g} \kappa_{g}^{\prime}}{\tau_{g}^{\prime} \kappa_{n}-\tau_{g} \kappa_{n}^{\prime}} e^{\frac{1}{2} \int \frac{\kappa_{g}\left(\kappa_{n}^{2}+\tau_{g}^{2}\right)^{\prime}-2 \kappa_{g}^{\prime}\left(\kappa_{n}^{2}+\tau_{g}^{2}\right)}{\kappa_{n}^{\prime} \tau_{g}-\tau_{g}^{\prime} \kappa_{n}} d s} . \\
& h=C_{3} e^{\frac{1}{2} \int \frac{\kappa_{g}\left(\kappa_{n}^{2}+\tau_{g}^{2}\right)^{\prime}-2 \kappa_{g}^{\prime}\left(\kappa_{n}^{2}+\tau_{g}^{2}\right)}{\kappa_{n}^{\prime} \tau_{g}-\tau_{g}^{\prime} \kappa_{n}} d s} \\
& =C_{1} \frac{\tau_{g} \kappa_{n}^{\prime}-\kappa_{n} \tau_{g}^{\prime}}{\kappa_{g}^{\prime} \kappa_{n}-\kappa_{g} \kappa_{n}^{\prime}} e^{\frac{1}{2} \int \frac{\tau_{g}\left(\kappa_{n}^{2}-\kappa_{g}^{2}\right)^{\prime}-2 \tau_{g}^{\prime}\left(\kappa_{n}^{2}-\kappa_{g}^{2}\right)}{\kappa_{g} \kappa_{n}^{\prime}-\kappa_{g}^{\prime} \kappa_{n}} d s} \\
& =C_{2} \frac{\tau_{g} \kappa_{n}^{\prime}-\kappa_{n} \tau_{g}^{\prime}}{\tau_{g} \kappa_{g}^{\prime}-\kappa_{g} \tau_{g}^{\prime}} e^{\frac{1}{2} \int \frac{\kappa_{n}\left(\kappa_{g}^{2}-\tau_{g}^{2}\right)^{\prime}-2 \kappa_{n}^{\prime}\left(\kappa_{g}^{2}-\tau_{g}^{2}\right)}{\kappa_{g} \tau_{g}^{\prime}-\tau_{g} \kappa_{g}^{\prime}} d s} .
\end{aligned}
$$

Thus the constant direction vector $V$ can be written as $V=f T+g Y+h Z$, where $V_{1}, V_{2}$, and $V_{3}$ are the above expressions. Thus from equations (39) and (42) we have

$$
\langle D, V\rangle=0 \text { and } V^{\prime}=0
$$


Note 14. The conditions for a spacelike curve with a timelike normal lying on a spacelike surface and a spacelike curve with a spacelike normal lying on a timelike surface to become a $K$-type slant helix can be calculated in the same way as we have done in the above sections using equations (5) and (6) in place of equations (3) and (8).

\section{Acknowledgements}

The first author is supported by UGC JRF of India, Ref. No. 1068/ CSIR NET JUNE 2019.

\section{References}

[1] A. T. Ali, Position vector of slant helix in Euclidean 3-space, J. Egyptian Math. Soc. 20 (2012), 1-6.

[2] A. T. Ali, R. López, and M. Turgut, K-type partially null and pseudo null slant helices in Minkowski 4-space, Math. Commun. 17 (2012), 93-103.

[3] A. T. Ali and L. Rafael, On slant helices in Minkowski space $E_{1}^{3}$, J. Korean Math. Soc. 48 (2011), 159- 167.

[4] A. T. Ali and M. Turgut, The position vector for timelike slant helix in $E_{1}^{3}$ space, J. Math. Anal. Appl. 365 (2010), 559-569.

[5] M. Barros, General helices and a theorem Lancret, Proc. Amer. Math. Soc. 125 (1997), 1503-1509.

[6] S. Izumiya and N. Takeuchi, New special curves and developable surfaces, Turk. J. Math. 28 (2004), 153-163.

[7] O. Kiliç and A. Çalişkan, The Frenet and Darboux instantaneous rotation vector for curves on spacelike surface, Math. Comput. Appl. 1(2) (1996), 77-86.

[8] L. Kula and Y. Yayli, On slant helix and its spherical indicatrix, Appl. Math. Comput. 169 (2005), 600-607.

[9] C. Y. Li, C. Zhu, and R. Wang, Spacelike developable surfaces through a common line of curvature in Minkowski 3-space, J. Adv. Mech. Design Syst. Manuf. 9(4) (2015), $9 \mathrm{pp}$.

[10] E. Nešović, U. Öztürk, and E. B. K. Öztürk, On k-type pseudo null Darboux helices in Minkowski 3-space, J. Math. Anal. Appl. 439 (2016), 690-700.

[11] U. Öztürk and E. Nešović, On pseudo null and null Cartan Darboux helices in Minkowski 3-space, Kuwait J. Sci. 43(2) (2016), pp. 64-82.

[12] U. Öztürk, E. Nešović, and E. B. K. Öztürk, On k-type spacelike slant helices lying on lightlike surfaces, Filomat 33(9) (2019), 2781-2796.

[13] H. H. Uğurlu and A. Topel, Relation between Darboux instantaneous rotation vector of curves on a time-like surface, Math. Comput. Appl. 1(2) (1996), 149-157.

[14] J. Walrave, Curves and Surfaces in Minkowski Space, Thesis (Ph.D.), Katholieke Universitiet Leuven (Belgium), 1995.

[15] E. Ziplar, A. Şenol and Y. Yayli, On Darboux helices in Euclidean 3-space, Glob. J. Sci. Front Res. 12 (2012), 72-80.

Department of Mathematics, Institute of Science, Banaras Hindu UniverSITY, VARANASI-221005, INDIA

E-mail address: thakursantoshbhu@gmail.com

E-mail address: pal.buddha@gmail.com 